\title{
Neural Comb Filtering using Sliding Window Attention Network for Speech Enhancement
}

\author{
P Venkatesh*, A Sivaganesh and K Sri Rama Murty
}

\begin{abstract}
In this paper, we demonstrate the significance of restoring harmonics of the fundamental frequency (pitch) in deep neural network (DNN) based speech enhancement. We propose a sliding-window attention network to regress the spectral magnitude mask (SMM) from the noisy speech signal. Even though the network parameters can be estimated by minimizing the mask loss, it does not restore the pitch harmonics, especially at higher frequencies. In this paper, we propose to restore the pitch harmonics in the spectral domain by minimizing cepstral loss around the pitch peak. The network parameters are estimated using a combination of the mask loss and cepstral loss. The proposed network architecture functions like an adaptive comb filter on voiced segments, and emphasizes the pitch harmonics in the speech spectrum. The proposed approach achieves comparable performance with the state-of-the-art methods with much lesser computational complexity ${ }^{1}$.
\end{abstract}

Index Terms-Spectral magnitude mask, transformer, pitch harmonics, cepstral pitch peak and production-related loss.

\section{INTRODUCTION}

Prolonged exposure to noisy speech signals causes severe fatigue to the listeners [1]. Speech Enhancement (SE) algorithms aim to improve the quality and intelligibility of the noisy speech signal, which is degraded by the background noise [2]. SE plays an important role in human-human communication over mobile/radio channels, hearing aids, and cochlear implants [3] etc. SE is a crucial first step for voice assistants in domestic environment having multiple noise sources like television, microwave oven, competing speakers etc. Even though several methods have been proposed in the literature, SE still remains a challenging problem, especially for unseen speakers and noises. SE algorithms can be broadly classified into two categories:

- Methods which rely on estimating the noise characteristics in order to suppress them.

- Methods which rely on estimating the speech characteristics in order to highlight them.

Most of the statistical SE algorithms rely on adaptive estimation of noise component and subtracting it from the noisy signal [4]-[6]. Deep neural network (DNN) approaches, on the other hand, rely on learning the structure of the speech signal through a non-linear mapping between noisy and clean speech signals [7]. As DNN approaches pose SE as a supervised learning task, a variety of noises at different signal-tonoise-ratios (SNRs) can be used during the training. Hence,

The authors are with Department of Electrical Engineering at Indian Institute of Technology Hyderabad, Hyderabad - 502285, Telangana, India. emails: parvathalavenkatesh123@gmail.com, ee18resch11020@iith.ac.in and ksrm@ee.iith.ac.in

${ }^{1}$ Audio samples: https://siplab-iith.github.io/SWAN
DNN approaches perform much better than the statistical approaches in unseen noises and lower SNR conditions [8], [9]. However, the superior performance of DNNs comes at the expense of huge computational complexity. There is a need to develop efficient low-complexity DNN architectures for lowpower applications [10].

Network architecture and loss function play an important role in efficiently capturing the signal characteristics. Convolutional and recurrent architectures have been commonly used in both time and frequency domains for SE [11]-[13]. However, the covolutional networks require more layers to increase the receptive field, while the recurrent networks are not suitable for parallel processing. Recent advances in transformer architectures offer compact models to capture longterm dependencies through explicit attention mechanism [14]. Kim et. al. proposed a Gaussian weighted self attention transformer (TGSA) for SE [15]. Subsequently, Wang et. al. proposed a two stage transformer neural network (TSTNN) in the time-domain for SE [16]. Although TSTNN is a compact model, it requires huge computation as it operates on every sample in the time-domain. In this paper, we propose a sliding window attention network (SWAN) to estimate spectral mask in the frequency domain. As the proposed architecture operates on the frames of speech signal, it requires significantly lesser computation than TSTNN.

The frequency domain approaches rely on estimating spectral mask from the noisy speech spectrum. The network parameters are updated using a combination of mask approximation loss and signal approximation loss [17]. State-of-theart frequency-domain approaches to SE use perception-related loss functions for signal approximation [18]-[20]. Although the perception related loss functions, operating in Mel/Bark domains, are good at approximating the lower frequency bands, they do not perform well in the high-frequency bands. In this paper, we propose a production-related loss function for signal approximation. Speech signal exhibits high SNR regions in both time and frequency domains. The region around the glottal-closure instants (GCIs) exhibit higher amplitudes in the time domain, and hence, they are less vulnerable to the degradation [21]. As periodicity of GCIs transforms to pitch harmonics in the frequency domain, adaptive comb-filtering has been used in the literature to enhance them [22], [23]. In this work, we propose a novel loss function to better approximate the pitch harmonics in the spectral mask estimated using a DNN. The estimated spectral mask resembles a comb-filter and enhances the noisy speech spectrum across the frequency bands. The rest of the paper is organized as follows: Section II presents proposed transformer architecture 
using sliding window. Production-related loss function to enhance pitch harmonics is discussed in Section III. Section IV provides a discussion on complexity vs performance tradeoffs of the SE algorithms. Finally, Section V summarizes the important contributions of this work.

\section{TRANSFORMER FOR SPECTRAL MASK Estimation}

In order to deal with the varying levels of degradation in the time-frequency (TF) plane, noisy signal is typically processed in the short-time Fourier transform (STFT) domain. The STFT of a noisy speech signal $s[n]$ can be expressed as

$$
S[t, k]=D[t, k]+G[t, k]
$$

where $t$ denotes frame index, $k$ denotes frequency bin, $D[t, k]$ and $G[t, k]$ are the STFTs of clean speech signal $d[n]$ and additive noise $g[n]$, respectively. In spectral masking approaches to $\mathrm{SE}$, the clean speech spectrum is estimated from the noisy speech spectrum as

$$
\hat{D}[t, k]=M[t, k] S[t, k]
$$

where $M[t, k]$ is the spectral mask. A detailed discussion on the spectral masks can be found in [7]. The spectral magnitude mask (SMM), given by

$$
M[t, k]=\frac{|D[t, k]|}{|S[t, k]|},
$$

has been shown to perform better [7]. The enhanced speech signal $\hat{d}[n]$ is reconstructed from $\hat{D}[t, k]$ using inverse STFT [24].

In this work, we used a transformer architecture shown in Fig. 1 to estimate SMM from $S[t, k]$. We have used timedistributed dense layers and temporal attention layers as the basic building blocks to facilitate the parallel processing of the data. The dense layers capture correlations across the frequency, while the temporal attention layers capture correlations across the time. The logarithm of the magnitude spectrum of the noisy speech signal is processed using a dense layer and batch-normalized to obtain context-independent nonlinear projections $\mathbf{x}[t]$. The sequence of context-independent projections are processed using a transformer block to extract the context information. As speech is a nonstationary signal, we have used sliding window attention to extract the context information [25]. The context vector estimated from the local temporal information is given by

$$
\mathbf{x}_{c}[t]=\mathbf{W}_{O} \sum_{\substack{\tau=t-c \\ \tau \neq t}}^{t+c} A[t, \tau] \mathbf{W}_{V} \mathbf{x}[\tau]
$$

where $\mathbf{W}_{0}$ and $\mathbf{W}_{V}$ are learnable linear transformations referred to as output and value projections respectively, $c$ is the temporal context, and $A[t, \tau]$ is the attention required from the frame- $\tau$ to enhance the frame- $t$. The attention weights are computed as

$$
A[t, \tau]=\operatorname{softmax}\left(\left(\mathbf{W}_{Q} \mathbf{x}[t]\right)^{\top} \mathbf{W}_{K} \mathbf{x}[\tau]\right),
$$

where $\mathbf{W}_{Q}$ and $\mathbf{W}_{K}$ are learnable linear transformations, referred to as query and key projections, respectively, and the

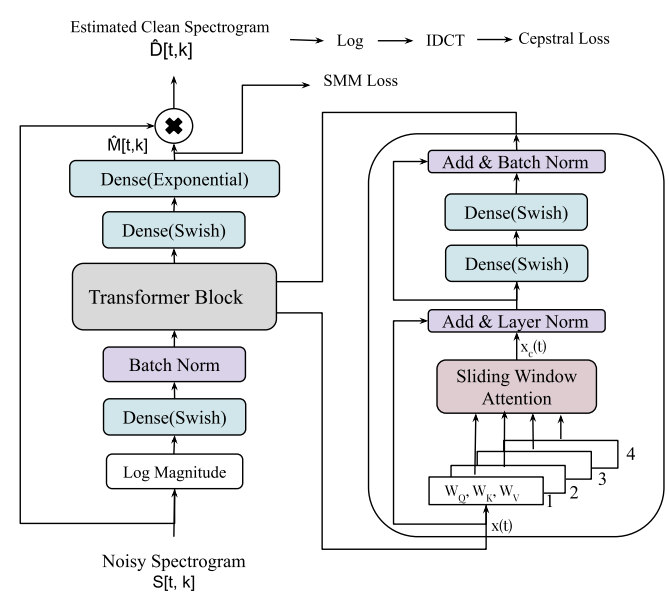

Fig. 1: Transformer architecture for estimating SMM

softmax function operates over the context window defined in (4). In the case of multihead attention, the context vector $\mathbf{x}_{c}[t]$ is estimated using multiple parallel projections as shown in Fig. 1. The context vector $\mathbf{x}_{c}[t]$ is added to the contextindependent vector $\mathbf{x}[t]$ through a residual connection and the sum is layer-normalized and processed using dense layers to capture correlations along frequency axis. When multiple layers of transformer blocks are employed, the signal is filtered along the time and frequency domains alternatively to estimate the SMM in the TF domain. The output of the transformer block is processed through a couple of fully connected layers to estimate the SMM. Assuming that the processing happens in log-spectral domain, an exponential activation function is employed in last layer to estimate the SMM. The concept of context vector of a transformer in (4) is familiar to the signal processing community. Similar operations are performed in spectral subtraction [4] and kernel adaptive filtering [26], with fixed weights and predetermined kernels, respectively. The recent advances in transformer architectures offer a way to learn optimum kernels/parameters from the data in a supervised manner. The network parameters can be estimated to minimize the distance between the oracle and estimated masks $\hat{M}[t, k]$

$$
L_{\text {mask }}=\frac{1}{|\mathcal{B}|} \sum_{u \in \mathcal{B}} \sum_{t} \sum_{k}\left|M^{u}[t, k]-\hat{M}^{u}[t, k]\right|_{p}
$$

where $u$ denotes utterance number, $|B|$ denotes cardinality of the batch, and $|\cdot|_{p}$ denotes $p$-norm of the error. The mean squared error (MSE) between the masks was used as the mask approximation objective [17]. However, closer observation of the oracle mask in Fig. 2(a) reveal occasional large amplitude fluctuations which need not be given a proportional weight in the error. Hence, we preferred to used mean absolute error (MAE), i.e., $\mathrm{p}=1$, as the mask approximation objective. In the earlier works, it has been reported that the mask approximation does not directly optimize the speech enhancement objective [17]. This could be because all the finer fluctuations in the oracle SMM may not be equally important for signal reconstruction. The objective function should help in prioritizing the fluctuations in SMM based 

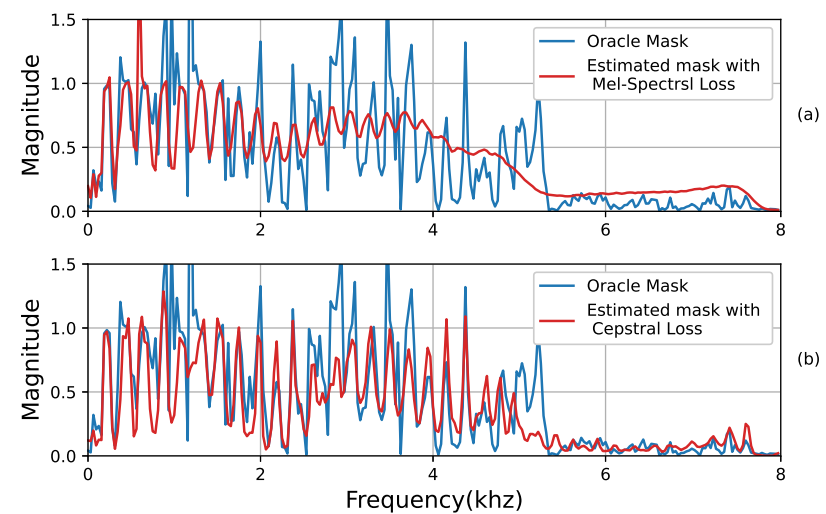

Fig. 2: Estimated SMM using (a) Mel-spectral loss, (b) Cepstral pitch-peak loss. Oracle mask is shown for comparison

on their significance in signal reconstruction. Hence a signal approximation objective is also used along with the mask approximation objective. Weninger [17] et. al., used MSE in the Mel-spectral domain as spectral approximation objective. The SMM estimated with this objective is shown in Fig. 2(a). While the estimated SMM matches well with the oracle mask in the low frequency bands $(<2 \mathrm{kHz})$, the SMM approximation is not good at the high frequency bands $(>2 \mathrm{kHz})$. In the subsequent studies, perceptual evaluation of speech quality (PESQ) was used as the signal approximation objective [19], [27]. These signal approximation objectives were motivated from the speech perception. Alternatively, the structure imposed by the speech production mechanism can be exploited for the signal reconstruction. In this work, we propose to exploit the quasi-periodic structure imposed by the voice source for speech enhancement.

\section{ENHANCING PITCH PEAK IN CEPSTRAL DOMAIN}

During the production of voiced sounds, significant excitation to the vocal-tract system is delivered at the GCIs [28]. Hence, the region around the GCI is more robust to the additive noise within each pitch period [21]. The quasiperiodic sequence of GCIs in time domain is manifested as pitch harmonics in the frequency domain, and as pitch peak in the cepstral domain [29]. The real cepstrum of clean speech signal can be computed from its spectrum $S[t, k]$ as

$$
S_{c}[t, q]=\left(\mathbf{W}_{D} \log |S[t, k]|\right)
$$

where $\mathbf{W}_{D}$ is the inverse discrete cosine transform (IDCT) matrix [30] and $q$ denotes quefrency bin in the cepstral domain. The lower quefrency band corresponds to the vocaltract information, while the higher quefrency band corresponds to the voice source information [31]. The most prominent peak at the cepstrum at high-time cepstrum corresponds to the pitch period [29]. Fig. 3(a) shows the cepstrum of a frame of clean speech signal collected from a female speaker, in which the peak at around $4.5 \mathrm{~ms}$ indicates the pitch period. The pitch peak retrieval was not good when Mel-spectral MSE is used as signal approximation loss as shown in 3(a).

The pitch harmonics can be enhanced in the spectrum by restoring pitch peak in the cepstral domain. In this work,
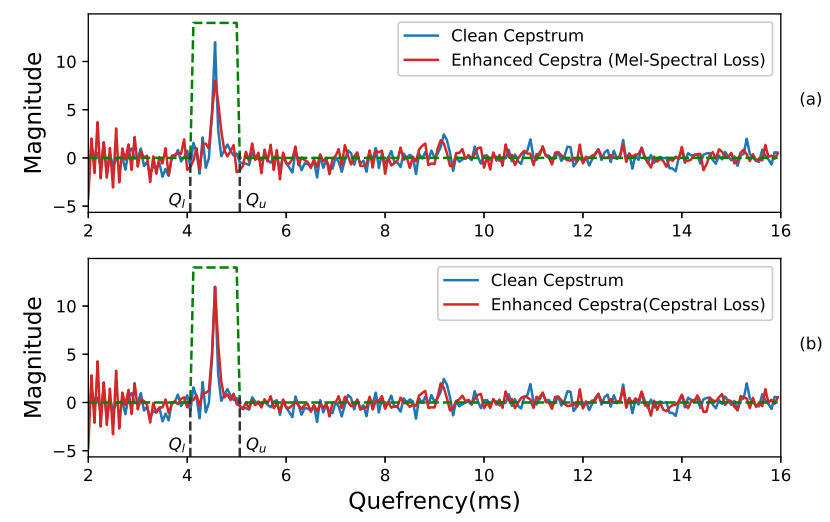

Fig. 3: Real cepstrum of a voiced frame of enhanced using (a) Mel-spectral loss, (b) Cepstral pitch-peak loss. Oracle cepstrum is shown for comparison

we propose to use the MSE between the pitch peaks of the clean and enhanced cepstra as signal approximation loss. The location of the pitch peak is predetermined using pitch tracking algorithm on clean speech data [32], [33]. In order to account for the minor deviations in pitch period estimation, we considered a short window around the expected pitch peak as shown in dotted lines of Fig. 3(a). The cepstral pitch-peak loss for a random batch $\mathcal{B}$ is computed as

$$
L_{c e p}=\frac{1}{|\mathcal{B}|} \sum_{u \in \mathcal{B}} \sum_{t} \sum_{q=Q_{l}}^{Q_{u}}\left|S_{c}^{u}[t, q]-\hat{S}_{c}^{u}[t, q]\right|
$$

where $\hat{S}_{c}[t, q]$ is the real-cepstrum of the enhanced signal, $Q_{l}$ and $Q_{u}$ denote the lower and upper bounds of the pitch window respectively. In this work $Q_{l}$ and $Q_{u}$ are chosen to be 7 samples each, to account for about $1 \mathrm{~ms}$ deviation in pitch period estimation. The composite loss function used for the network training is given by

$$
L=\alpha L_{m a s k}+(1-\alpha) L_{c e p} .
$$

where the optimum value for $\alpha(=0.9)$ is determined empirically using grid search. Fig. 3(b) illustrates the effectiveness of the proposed production-related loss function in retrieving the pitch peak. As a consequence, the estimated SMM in Fig. 2(b) resembles a comb-filter enhancing pitch harmonics in magnitude spectrum.

\section{EXPERIMENTAL EVALUATION}

The performance of the proposed sliding window attention network (SWAN) is evaluated on publicly available speech dataset [37] which contains 11572 utterances from 28 speakers (14 male +14 female) for training and 824 utterances from 2 speakers ( 1 male +1 female) for testing. The noisy samples are prepared by mixing 10 different noises ( 8 from DEMAND dataset [38] and 2 artificially generated noises) at SNRs $15 \mathrm{~dB}$, $10 \mathrm{~dB}, 5 \mathrm{~dB}$ and $0 \mathrm{~dB}$ for training, and 5 different unseen noises at 4 SNRs $17.5 \mathrm{~dB}, 12.5 \mathrm{~dB}, 7.5 \mathrm{~dB}$ and $2.5 \mathrm{~dB}$ for testing. All the utterances are downsampled to $16 \mathrm{kHz}$.

Speech segments of length of $1.616 \mathrm{~s}$ are randomly sliced from the utterances, and STFT is computed with a frame size of $32 \mathrm{~ms}$, shifted by $16 \mathrm{~ms}$, resulting in 100 frames per 
TABLE I: Performance comparison of SWAN with state-of-the-art methods

\begin{tabular}{lcccccccrr}
\hline Model & Domain & PESQ & STOI & CSIG & CBAK & COVL & SSNR & Parameters(M) & GFLOPS \\
\hline Noisy & - & 1.97 & 92.0 & 3.35 & 2.44 & 2.63 & 1.68 & - & - \\
Weiner & - & 2.22 & 93.0 & 3.23 & 2.68 & 2.67 & 5.07 & - & - \\
DEMUCS [34] & T & 3.04 & 95.3 & 4.36 & 3.52 & 3.72 & 9.28 & 33.5 & 137.00 \\
TSTNN [35] & T & 2.94 & 94.7 & 4.30 & 3.54 & 3.64 & 9.93 & 0.92 & 43.10 \\
Metricgan+ [36] & TF & 3.13 & 93.2 & 4.10 & 2.90 & 3.61 & 3.76 & 1.89 & 0.25 \\
\hline SWAN(1L) & TF & 2.90 & 93.70 & 4.06 & 3.38 & 3.48 & 8.22 & 0.53 & 0.08 \\
SWAN(3L) & TF & 2.95 & 94.18 & 4.13 & 3.44 & 3.55 & 8.62 & 1.19 & 0.17 \\
SWAN(6L) & TF & 2.99 & 94.23 & 4.13 & 3.47 & 3.57 & 8.83 & 2.18 & 0.30 \\
\hline SWAN(1L, Causal) & TF & 2.80 & 93.38 & 3.97 & 3.31 & 3.39 & 7.94 & 0.53 & 0.08 \\
SWAN(3L, Causal) & TF & 2.84 & 93.74 & 4.01 & 3.38 & 3.43 & 8.61 & 1.19 & 0.16 \\
SWAN(6L, Causal) & TF & 2.85 & 93.75 & 4.03 & 3.39 & 3.44 & 8.70 & 2.18 & 0.29 \\
\hline
\end{tabular}

segment. The network is trained to estimate SMM from the 257-dimensional magnitude spectra. All the dense layers in the network are of 257 dimensions. In the transformer block we have used 4-heads with 64-dimensional linear projections. We have used the same linear projection values and keys, i.e., $\mathbf{W}_{K}=\mathbf{W}_{V}$. A sliding window of 21 frames $(c=10)$ is used to evaluate the context vector in (4). The network is trained to minimize the composite loss function in (9), using Adam optimizer [39], with a batch size of 8 segments. The pitch peak location required for loss computation is estimated from the clean speech signal using YAAPT algorithm [32]

The proposed method is compared with the three stateof-the-art methods in the literature, viz., DEMUCS [40], TSTNN [16] and Metricgan+ [20], for which either the codes or pretrained models are available online [34]-[36]. DEMUCS and TSTNN are time-domain approaches while Metrigan+ is a frequency domain approach. DEMUCS uses a combination of convolutional and long short term memory (LSTM) layers, while the TSTNN uses a combination of convolutional and transformer layers. However, the transformer layer of TSTNN consists of a gated recurrent unit (GRU), instead of conventional dense layer. Both DEMUCS and TSTNN are trained to minimize a combination of waveform reconstruction loss and STFT loss. Metricgan+ is a generative adversarial network in which the generator consisting of bidirectional LSTM (BLSTM) layers estimates the SMM, and the discriminator consisting convolutional layers evaluates the perceptual quality of the enhanced signal. The discriminator of the Metricgan+ was designed to mimic the behavior of PESQ metric.

The performance of the SE algorithms is evaluated using the following objective quality measures: perceptual evaluation of speech quality (PESQ) [41], short-time objective intelligibility (STOI) [42], objective correlates of mean opinion scores [43] which include signal distortion (CSIG), background noise intrusion (CBAK) and overall quality evaluation (COVL), and segmental SNR (SSNR). In addition, we have evaluated the computational complexity of different algorithms in terms of gillions of floating point operations [44] (GFLOPs) required to enhance one second of speech segment.

Table I compares the performance of SWAN with the stateof-the-art SE methods. The performance of SWAN is evaluated with different number of transformer blocks to understand the model complexity vs. performance tradeoff. The number of model parameters is also listed in the Table I. Metricgan+ achieves the highest PESQ of 3.13 as it is trained to optimize the PESQ metric explicitly. However, its STOI and SSNR suffered because of the time-varying gain introduced while optimizing the PESQ metric. As DEMUCS and TSTNN are time-domain approaches, they perform better on these metrics. However, the computational complexity of the time-domain approaches is very high as they operate on every sample in the speech signal. On the other hand, frequency domain approaches like Metricgan+ and SWAN operate on signal frames, and hence, require lesser computation. In particular, SWAN offers a good balance between the enhancement quality and the computational complexity. The performance of SWAN is comparable to the time-domain approaches across the metrics, even it requires less than one-hundredth of the computation. As the SWAN is trained to minimize the productionrelated loss function, its SSNR and STOI are better than the Metricgan+. Even a single-layer SWAN, with just $0.53 \mathrm{M}$ parameters, delivers an impressive performance illustrating the effectiveness of the proposed production-related loss function for speech enhancement. The sliding-window attention can be restricted to include only the causal context for enhancing streaming audio in real-time. However, the performance is slightly compromised with causal attention. Among the stateof-the-art methods in Table I, only DEMUCS can be applied on streaming audio. The other two methods require the entire signal for processing.

The proposed approach enhances only the magnitude spectrum of the speech signal, and reuses the noisy phase during reconstruction. In future, it will be interesting to develop mechanisms to include phase into context vector generation. The performance can be improved by combining the proposed production-related loss function with the perception-related loss function.

\section{Summary AND CONCLUSION}

While end-to-end architectures with general purpose loss functions are attractive, incorporating domain-specific knowledge can significantly reduce the network complexity and improve the interpretability. This work demonstrates the importance of incorporating production-related information in DNN training for speech enhancement. The proposed SWAN architecture is trained to minimize a combination of mask loss and cepstral loss around the pitch peak. The spectral mask thus estimated resembles a comb-filter emphasizing the high SNR regions around the pitch harmonics. 


\section{REFERENCES}

[1] M. Klatte, T. Lachmann, and M. Meis, "Effects of noise and reverberation on speech perception and listening comprehension of children and adults in a classroom-like setting," Noise and Health, vol. 12, no. 49, 2010.

[2] P. Loizou, Speech enhancement: theory and practice. CRC press, 2007.

[3] L.-P. Yang and Q.-J. Fu, "Spectral subtraction-based speech enhancement for cochlear implant patients in background noise," The Journal of the Acoustical Society of America, vol. 117, no. 3, 2005.

[4] S Boll, "Suppression of acoustic noise in speech using spectral subtraction," IEEE Transactions on speech signal, 1979.

[5] P. Scalart and J. V. Filho, "Speech enhancement based on a priori signal to noise estimation,' in ICASSP, IEEE International Conference on Acoustics, Speech and Signal Processing - Proceedings, vol. 2, 1996.

[6] Y. Ephraim and D. Malah, "Speech Enhancement Using a Minimum Mean-Square Error Short-Time Spectral Amplitude Estimator," IEEE Transactions on Acoustics, Speech, and Signal Processing, vol. 32, no. 6, 1984.

[7] D. Wang and J. Chen, "Supervised speech separation based on deep learning: An overview," in IEEE/ACM Transactions on Audio Speech and Language Processing, vol. 26, no. 10, 2018.

[8] S. W. Fu, Y. Tsao, and X. Lu, "SNR-aware convolutional neural network modeling for speech enhancement," in Proceedings of the Annual Conference of the International Speech Communication Association, INTERSPEECH, September 2016.

[9] S. R. Park and J. W. Lee, "A fully convolutional neural network for speech enhancement," in Proceedings of the Annual Conference of the International Speech Communication Association, INTERSPEECH, August 2017.

[10] M. Romaniuk, P. Masztalski, K. Piaskowski, and M. Matuszewski, "Efficient low-latency speech enhancement with mobile audio streaming networks," in Proceedings of the Annual Conference of the International Speech Communication Association, INTERSPEECH, October 2020.

[11] Y. Luo and N. Mesgarani, "Conv-TasNet: Surpassing Ideal TimeFrequency Magnitude Masking for Speech Separation," IEEE/ACM Transactions on Audio Speech and Language Processing, vol. 27, no. 8, 2019.

[12] P. S. Rani, S. Andhavarapu, and S. R. Murty Kodukula, "Significance of Phase in DNN based speech enhancement algorithms," in 26th National Conference on Communications, NCC, 2020.

[13] S. Pascual, A. Bonafonte, and J. Serra, "SEGAN: Speech enhancement generative adversarial network," in Proceedings of the Annual Conference of the International Speech Communication Association, INTERSPEECH, August 2017.

[14] A. Vaswani, N. Shazeer, N. Parmar, J. Uszkoreit, L. Jones, A. N. Gomez, L. Kaiser, and I. Polosukhin, "Attention is all you need," in Advances in Neural Information Processing Systems, December 2017.

[15] J. Kim, M. El-Khamy, and J. Lee, "T-GSA: Transformer with GaussianWeighted Self-Attention for Speech Enhancement," in IEEE International Conference on Acoustics, Speech and Signal Processing (ICASSP), May 2020.

[16] K. Wang, B. He, and W.-P. Zhu, "TSTNN: Two-Stage Transformer Based Neural Network for Speech Enhancement in the Time Domain," in IEEE International Conference on Acoustics, Speech and Signal Processing (ICASSP), 2021.

[17] F. Weninger, J. R. Hershey, J. Le Roux, and B. Schuller, "Discriminatively trained recurrent neural networks for single-channel speech separation," in IEEE Global Conference on Signal and Information Processing, GlobalSIP, 2014.

[18] Q. Wang, J. Du, L.-R. Dai, and C.-H. Lee, “A multiobjective learning and ensembling approach to high-performance speech enhancement with compact neural network architectures," IEEE/ACM Transactions on Audio, Speech, and Language Processing, vol. 26, no. 7, pp. 11851197, 2018.

[19] J. Kim and M. El-Kharmy, "End-to-end multi-task denoising for joint SDR and PESQ optimization," arXiv:1901.09146, J Lee - arXiv preprint, 2019.

[20] S.-W. Fu, C. Yu, T.-A. Hsieh, P. Plantinga, M. Ravanelli, X. Lu, and Y. Tsao, "Metricgan+: An improved version of metricgan for speech enhancement," arXiv preprint arXiv:2104.03538, 2021.

[21] B. Yegnanarayana, C. Avendano, H. Hermansky, and P. S. Murthy, "Speech enhancement using linear prediction residual," Speech communication, vol. 28, no. 1, pp. 25-42, 1999.

[22] D. Malah and R. Cox, "A generalized comb filtering technique for speech enhancement," in ICASSP'82. IEEE International Conference on Acoustics, Speech, and Signal Processing, vol. 7. IEEE, 1982, pp. $160-163$.

[23] A. Nehorai and B. Porat, "Adaptive comb filtering for harmonic signal enhancement," IEEE Transactions on Acoustics, Speech, and Signal Processing, vol. 34, no. 5, pp. 1124-1138, 1986.

[24] R. Crochiere, "A weighted overlap-add method of short-time fourier analysis/synthesis," IEEE Transactions on Acoustics, Speech, and Signal Processing, vol. 28, no. 1, pp. 99-102, 1980.

[25] I. Beltagy, M. E. Peters, and A. Cohan, "Longformer: The longdocument transformer," arXiv preprint arXiv:2004.05150, 2020.

[26] W. Liu, J. C. Principe, and S. Haykin, Kernel Adaptive Filtering: A Comprehensive Introduction. John Wiley \& Sons, 2010.

[27] S.-W. Fu, C.-F. Liao, Y. Tsao, and S.-D. Lin, "Metricgan: Generative adversarial networks based black-box metric scores optimization for speech enhancement," in International Conference on Machine Learning. PMLR, 2019, pp. 2031-2041.

[28] K. S. R. Murty and B. Yegnanarayana, "Epoch extraction from speech signals," IEEE Transactions on Audio, Speech, and Language Processing, vol. 16, no. 8, pp. 1602-1613, 2008.

[29] A. M. Noll, "Cepstrum Pitch Determination," The Journal of the Acoustical Society of America, vol. 41, no. 2, 1967.

[30] K. Rao and P. Yip, Discrete cosine transform: algorithms, advantages, applications. Academic press, 2014.

[31] L. Rabiner, Fundamentals of speech recognition. PTR Prentice Hall, 1993.

[32] K. Kasi and S. A. Zahorian, "Yet another algorithm for pitch tracking," in IEEE international conference on acoustics, speech, and signal processing, vol. 1. IEEE, 2002, pp. I-361.

[33] S. A. Zahorian and H. Hu, "A spectral/temporal method for robust fundamental frequency tracking," The Journal of the Acoustical Society of America, vol. 123, no. 6, 2008.

[34] A. Defossez, G. Synnaeve, and Y. Adi, "DEMUCS implementation codes and Pre-trained models," https://github.com/facebookresearch/ denoiser.

[35] K. Wang, B. He, and W.-P. Zhu, "TSTNN implementation codes," https: //github.com/key2miao/TSTNN.

[36] S.-W. Fu, C. Yu, T.-A. Hsieh, P. Plantinga, M. Ravanelli, X. Lu, and Y. Tsao, "Metricgan+ pre-trained model and inference function," https: /huggingface.co/speechbrain/metricgan-plus-voicebank.

[37] C. Valentini-Botinhao, X. Wang, S. Takaki, and J. Yamagishi, "Investigating rnn-based speech enhancement methods for noise-robust text-tospeech." in SSW, 2016, pp. 146-152.

[38] J. Thiemann, N. Ito, and E. Vincent, "The diverse environments multichannel acoustic noise database: A database of multichannel environmental noise recordings," The Journal of the Acoustical Society of America, vol. 133, no. 5, 2013.

[39] D. P. Kingma and J. L. Ba, "Adam: A method for stochastic optimization," in 3rd International Conference on Learning Representations, ICLR - Conference Track Proceedings, 2015.

[40] A. Défossez, G. Synnaeve, and Y. Adi, "Real time speech enhancement in the waveform domain," in Proceedings of the Annual Conference of the International Speech Communication Association, INTERSPEECH, October 2020

[41] I.-T. Recommendation, "Perceptual evaluation of speech quality (PESQ): An objective method for end-to-end speech quality assessment of narrow-band telephone networks and speech codecs," Rec. ITU-T P. 862, 2001.

[42] C. H. Taal, R. C. Hendriks, R. Heusdens, and J. Jensen, "A shorttime objective intelligibility measure for time-frequency weighted noisy speech," in ICASSP, IEEE International Conference on Acoustics, Speech and Signal Processing - Proceedings, 2010.

[43] Y. Hu and P. C. Loizou, "Evaluation of objective quality measures for speech enhancement," IEEE Transactions on Audio, Speech and Language Processing, vol. 16, no. 1, 2008.

[44] D. Terpstra, H. Jagode, H. You, and J. Dongarra, "Collecting performance data with PAPI-C," in Proceedings of the 3rd International Workshop on Parallel Tools for High Performance Computing 2009, 2010 . 